\title{
P02.43. A Mediterranean-style, low-glycemic diet plus phytonutrient rich medical food improves cardiovascular risk variables in women with metabolic syndrome
}

\author{
R Lerman ${ }^{1 *}$, M Mclntosh $^{2}$, M Fernandez ${ }^{3}$, W Najm $^{4}$ \\ From International Research Congress on Integrative Medicine and Health 2012 \\ Portland, Oregon, USA. 15-18 May 2012
}

\section{Purpose}

Metabolic syndrome (MetS) is a growing public health concern and effective dietary intervention programs could make a tremendous impact on slowing disease progression. To assess the benefits of a dietary program on cardiometabolic diease risk variables, a 12-week, randomized controlled trial of overweight and obese women with MetS was conducted.

\section{Methods}

Participants consumed a Mediterranean-style, low-glycemic-load diet (control arm, $\mathrm{n}=44$ ), or the same diet plus a medical food (UltraMeal PLUS 360, Metagenics Inc.) containing phytosterols, soy protein, and extracts from hops and acacia (intervention arm, $\mathrm{n}=45$ ). Fasting blood samples were analyzed at baseline, week 8 , and week 12 for plasma lipids, apolipoproteins, and homocysteine. Dietary records were collected and analyzed.

\section{Results}

Reduction in fat and sugar intake ( $\mathrm{p}<.001$ for both) was observed and increases in docosahexaenoic acid and eicosapentaenoic acid intake ( $\mathrm{p}<.001$ for both) were recorded, consistent with the prescribed diet. Regarding MetS variables, decreases in waist circumference, systolic and diastolic blood pressure, and plasma triglycerides in all subjects ( $\mathrm{p}<.001$ for all) were observed, with no differences between arms. Plasma low-density lipoprotein cholesterol, non-high-density lipoprotein cholesterol, apolipoprotein (apo) B, and apo B/apo A1 were reduced over the 12-wk study, but to a greater extent in the intervention arm ( $\mathrm{p}<.05$ for all), indicating the medical food had an effect in altering lipoprotein metabolism. Further, medical food intake was associated with reduced plasma homocysteine ( $\mathrm{p}<.01)$, compared to the control arm.

\section{Conclusion}

A Mediterranean-style, low-glycemic-load diet effectively reduced cardiovascular risk factors associated with MetS. Addition of medical food resulted in an improved lipoprotein profile and lowered plasma homocysteine.

\section{Author details}

${ }^{1}$ Metagenics Inc., Gig Harbor, USA. ²Department of Emergency Medicine, University of Florida, Jacksonville, Jacksonville, USA. ${ }^{3}$ Department of Nutritional Sciences, University of Connecticut, Storrs, USA. ${ }^{4}$ Department of Medicine, University of California, Irvine, Irvine, USA.

Published: 12 June 2012

doi:10.1186/1472-6882-12-S1-P99

Cite this article as: Lerman et al:: P02.43. A Mediterranean-style, lowglycemic diet plus phytonutrient rich medical food improves cardiovascular risk variables in women with metabolic syndrome. BMC Complementary and Alternative Medicine 2012 12(Suppl 1):P99. 